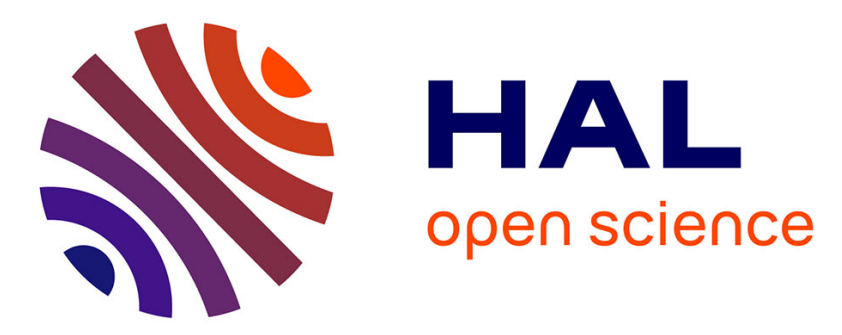

\title{
Experimental power transfer and signal assessment of wireless communications in reverberating cavities
}

\author{
Wilfrid Quenum, Isabelle Junqua, Jean-Philippe Parmantier
}

\section{To cite this version:}

Wilfrid Quenum, Isabelle Junqua, Jean-Philippe Parmantier. Experimental power transfer and signal assessment of wireless communications in reverberating cavities. 2016 IEEE Wireless Power Transfer Conference (WPTC), 2016, Aveiro, Portugal. 10.1109/WPT.2016.7498796 . hal-01436390

\section{HAL Id: hal-01436390 \\ https://hal.science/hal-01436390}

Submitted on 16 Jul 2021

HAL is a multi-disciplinary open access archive for the deposit and dissemination of scientific research documents, whether they are published or not. The documents may come from teaching and research institutions in France or abroad, or from public or private research centers.
L'archive ouverte pluridisciplinaire HAL, est destinée au dépôt et à la diffusion de documents scientifiques de niveau recherche, publiés ou non, émanant des établissements d'enseignement et de recherche français ou étrangers, des laboratoires publics ou privés. 


\title{
Experimental power transfer and signal assessment of wireless communications in reverberating cavities
}

\author{
Wilfrid.Quenum ${ }^{1}$, Isabelle Junqua ${ }^{1}$, Jean Philippe Parmantier ${ }^{1}$, \\ ${ }^{1}$ DEMR - UFTMiP - ONERA, The French Aerospace Lab, Toulouse, France, wilfrid.quenum@onera.fr
}

\begin{abstract}
This paper focuses on experimental measurement of power and signal transfer of wireless communication in reverberating cavities. The experiment is based on the evaluation of a transfer function between two antennas placed inside and outside a confined reverberating environment made of several metallic cavities. Inside these cavities, a WIFI communication is used in order to control the measurement setup in order to take advantage of its simple connection with the measurement devices. Therefore the first exploitation of this experiment is directly to measure S-parameters as main parameters for characterizing this transfer functions. The second type of exploitation consists in the assessment of the integrity of the WIFI communication in such a closed and confined reverberating environment.
\end{abstract}

Key words: Wireless communication; Transfer functions, Mode Stirred Reverberating Chambers, MSRC, WIFI

\section{INTRODUCTION}

Wireless deployment in various nowadays system technologies has become an interesting challenge to simplify the communication with electronic equipment. For example some aircraft applications are interested in replacing wireline networks by robust and safe wireless solutions to either reduce maintenance on cables or to implement wireless communications in areas which are not easily accessible. Further on, new trends of wireless exploitation try to investigate other types of applications of wireless links; this is for example the case for search of opportunities to exploit the energy budget in wireless power transmissions (WPT) for which one part of the transmitted signal is split for the channel communication and the other part for energy supply [1].

Whatever these new wireless communications links, they must of course fulfil classical EMC and Telecommunication standards [2]; particularly they must comply with accepted emission power of the wireless systems.

Amongst wireless solutions, WIFI is the most popular known even if it is not the most secure. This is the reason why other spectral bands and protocols are under study to ensure signal integrity especially for critical functions with stringent safety considerations as can be found in aeronautics.

A key factor of performance of such secure wireless communication is the quality of the propagation channel which must be precisely characterized and controlled. In this context, reverberating cavities, initially developed for Electromagnetic Compatibility (EMC) purposes for testing the susceptibility of equipment to electromagnetic (EM) environments, have been used to experimentally model and evaluate the performance of typical propagation channels for indoors and outdoors applications of wireless links under development ([3], [4], [5]).

The work presented in this paper intends

- By exploiting the properties of reverberating enclosures to develop an experimental set-up in order to characterize a propagation channel in small, closed and confined geometrical environments such as in aircraft bays, by using a WIFI communication

- To evaluate the integrity of WIFI signals in such environments.

\section{EXPERIMENTAL SETUP}

\section{A. Description of the structure under test}

The structure under study is a large laboratory metallic parallelepiped cavity of about $2 \mathrm{~m}^{3}$ made of three connected elementary metallic cavities with the following dimensions: length $1 \mathrm{~m}, 0.9 \mathrm{~m}$, height $0.70 \mathrm{~m}$. This structure is classically used as a Mode Stirring Reverberating Chamber (MSRC) for EMC applications for frequencies above $1 \mathrm{GHz}$. Some walls can be inserted between the three cavities in order to obtain smaller interconnected inner volumes. The separating walls can have several apertures in order to make possible the transmission of EM signals. It is equipped with a step-by-step stirrer located on one of the 3 cavities. It has also different trapdoors which can be opened and or closed with panel features allowing opening simulation or connection of antennas as illustrated in Figure 1.

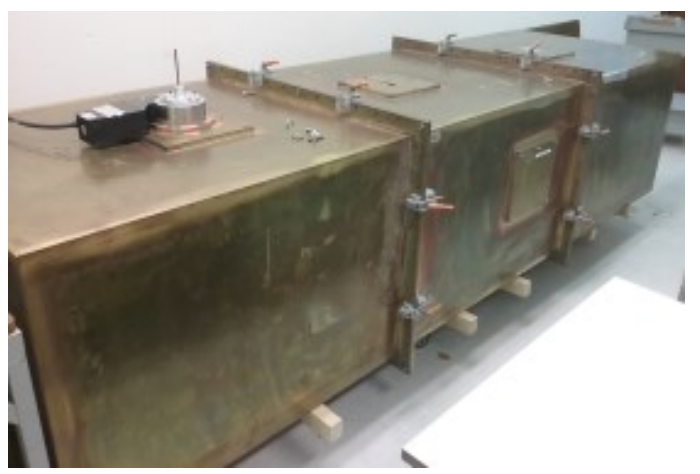

Figure 1: The three reverberating cavities and the stirrer electrical motor on the top side of one cavity 


\section{B. Measurement setup}

The experiment consists in measuring the transfer function (TF) between two antennas (horn-antennas) placed at two extremities of the structure (front and rear panels). The TF is measured with a network analyser as a $\mathrm{S}_{21}$-parameter between $1 \mathrm{GHz}$ and $6 \mathrm{GHz}$. The network analyser, and the measurement cables are outside the structure and a full 2-port calibration is made at the extremities of the measurement cables.

Therefore, the TF measured with a network analyser is stored for each position of the stirrer, controlled by step-bystep electrical motor. The motor is driven by a PC. 36 positions over a complete rotation of the stirrer are classically considered to provide the maximum number of "independent" positions in this measurement environment.

Finally, as classically done in MSRCs in EMC, the TF measurements are post-processed in order to get the statistical behaviour over a complete stirrer rotation. The statistical mean value is defined as (1) where $\mathrm{N}$ is the total number of stirrer positions on a $360^{\circ}$ rotation (here $\mathrm{N}=36$ ):

$$
\left\langle\left|S_{21}\right|^{2}\right\rangle=\frac{1}{N} \sum_{i=1}^{N}\left|S_{21}^{i}\right|^{2}
$$

Considering that antennas are placed in the cavity so that the direct coupling can be neglected, (1) informs on the properties of reverberation in the enclosure [6]. It depends on the total energy budget between the 2 antennas which depends itself on the dissipative losses inside the structure. At the same time, this mean values finally enables characterization of the performances of the wireless communication channel.

One objective being to analyse how a WIFI communication protocol can be used to drive the whole experimentation, two configurations have been considered as a first step of the experiment:

- A reference configuration: the network analyser and the motor of the stirrer are driven by the PC through a GPIB cable link.

- A WIFI configuration: the PC drives the instrumentation through a WIFI link as illustrated in Figure 2.

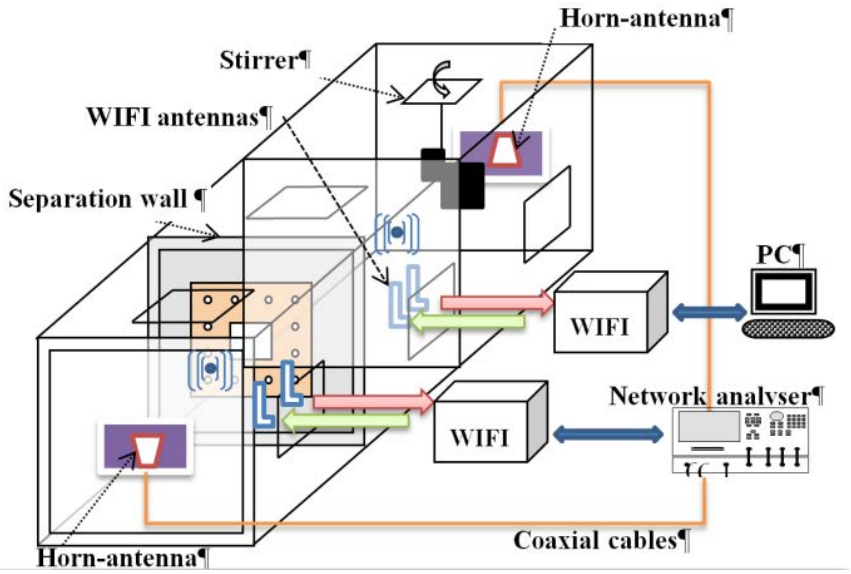

Figure 2: Complete setup synoptic with the WIFI measurement link

Without going into the details of the WIFI protocol [1], the WIFI system used is a dual band D-Link integrated system which enables us to change the power delivered and brief access to the frame and data channel statistics. In the experiment, the WIFI frequency is fixed at $5.26 \mathrm{GHz}$ and the WIFI antennas are mounted either inside (Figure 3 ) or outside the structure.

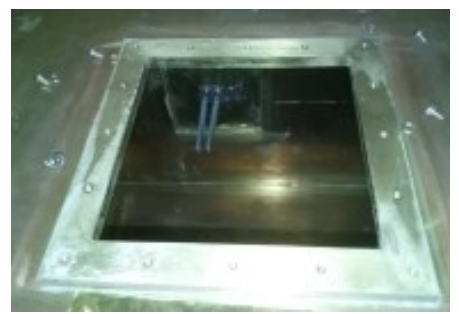

Figure 3: Dual WIFI antenna pair inside one cavity

\section{TRANSFER FUNCTION MEASUREMENT}

Here, the objective is to evaluate the impact of a WIFI communication and its possible interference onto the hornantenna link. For this the TF measurement between the two horns antennas, obtained in the reference configuration (GPIB) and in the various WIFI configurations are compared.

The enclosure is constituted of the full volume without any walls between the elementary cavities. Two WIFI measurement configurations have been considered: both WIFI antennas have been placed inside the structure (WIFI INSIDE), both WIFI antennas have been placed outside the structure (WIFI OUTSIDE) in order to isolate the WIFI measurement link from the TF link between the two horn antennas. The WIFI power source has been set at its maximum $(18 \mathrm{dBm})$. Figure 4 shows this comparison on the whole frequency band $(1 \mathrm{GHz}-6 \mathrm{GHz})$.

As seen in Figure 4, the TF is the same in all three configurations which proves the total independency of the transfer link between the two horn antennas and the WIFI communication link. Consequently, an interesting conclusion is that a power source of $18 \mathrm{dBm}$ is sufficient to provide the WIFI measurement link a sufficient sensibility to detect low level signals

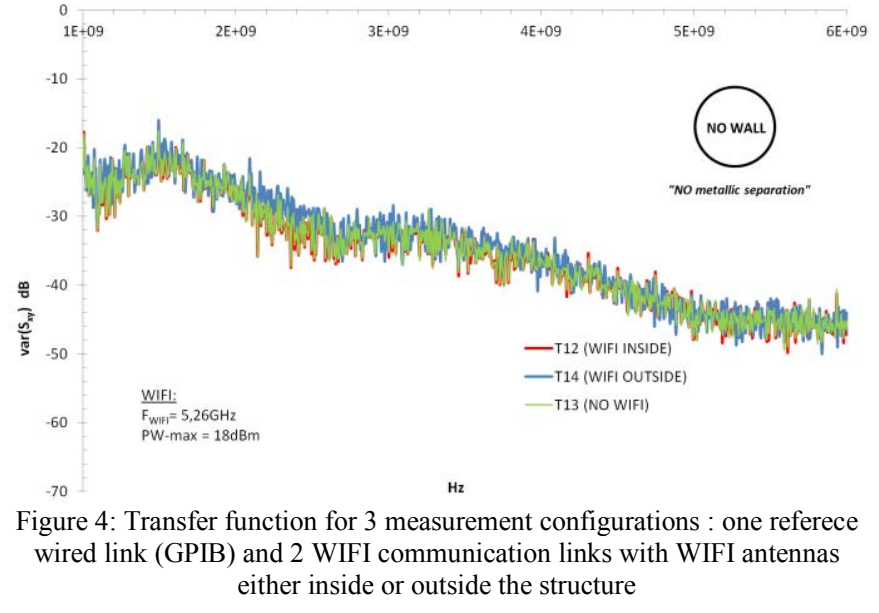

\section{WIFI INTEGRITY}

As already mentioned in the objectives, we are also interested in evaluating the integrity of the WIFI signal. 
In order to artificially and significantly alter the propagation channel (for both wireless and WIFI links), we have introduced in the enclosure a separating wall (WALL 1) equipped with a small circular aperture which divides the total enclosure in two volumes as shown in Figure 5. In this case, both WIFI and horn-antenna propagation paths will be attenuated due to the transfer through this aperture.

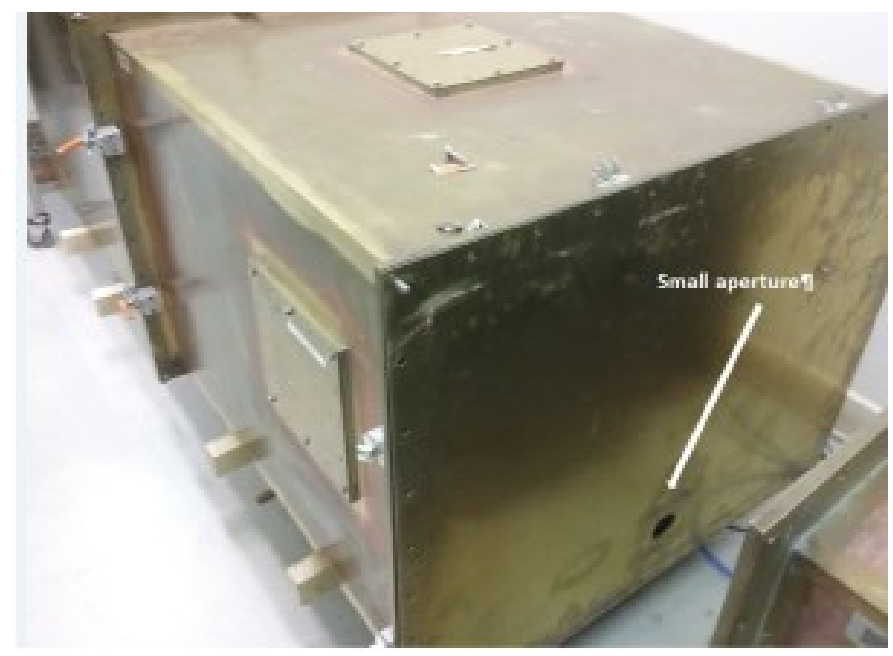

Figure 5: Separating metallic wall equipped with a small communication aperture

\section{A. Power and frequency spectrum of EM environment}

In this configuration, we consider, here, that both WIFI antennas and both horn-antennas are placed inside the structure, each one in one of the two volumes. The objective is now to measure with a dipole antenna and a spectrum analyzer (in "max hold" position) the power spectrum of the EM environment inside the receiving enclosure (the enclosure containing the receiving horn-antenna) on the $1 \mathrm{GHz} 6 \mathrm{GHz}$ bandwidth. In Figure 6, the spectrum is measured in 3 configurations:

- Configuration WIFI3: the WIFI communication link is activated alone with a power source of $18 \mathrm{dBm}$,

- the wireless antenna-horn link and the WIFI communication are activated simultaneously with respectively a WIFI source power of $18 \mathrm{dBm}$ (configuration TF AS-T16) and the minimal possible WIFI source power $(<9 \mathrm{dBm})$ (configuration TF-AS-T17)

In the case of simultaneous activation of horn-antenna and WIFI the measured spectrum being set in hold positon always shows the global spectrum of all the samples of stirrer positions, over the full $360^{\circ}$ rotation. As expected, it is possible to identify on the one hand, the spectral lines due to the WIFI signal (and especially the main line at $5.86 \mathrm{GHz}$ ) and, on the other hand, the spectral lines of the network analyzer on the receiving horn antenna due. The discrete highly sampled TF is due to the altered WIFI measurement transmission in the enclosure. Note that this alteration is obtained for the two values of the WIFI power sources; the not-corrupted lines with the lowest power are not necessarily not-corrupted lines for the maximum power .

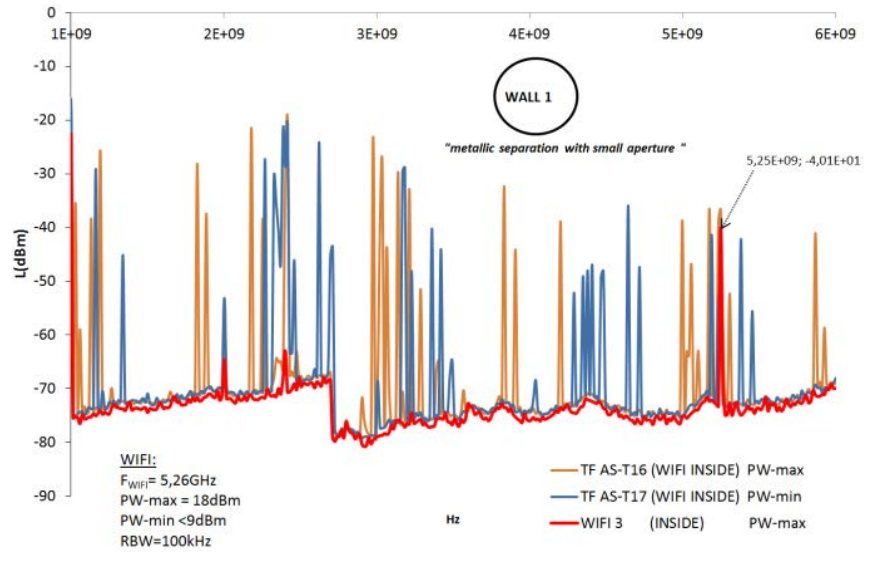

Figure 6 : Power spectrum inside the receiving cavity during TF measurement (blue and brown) and WIFI alone (in red)

\section{B. Path attenuation and WIFI integrity}

Now the transfer function (TF) between the two horn antennas located in the 2 different elementary volumes of the enclosure are measured with the WIFI communication link (Figure 7). Three configurations have been considered:

- Reference GPIB configuration: experimental set-up with the GPIB link,

- INSIDE WIFI configuration: both WIFI antennas are inside the structure in each of the two volumes and the source power is either maximal or minimal,

- IN-OUT WIFI configuration: a WIFI antenna is inside the structure and the other WIFI antenna is outside the structure. Only a minimal WIFI power source is used

For the WIFI configurations, the transfer function is either the statistical mean over a complete rotation of the stirrer defined as in section 1 ("36POS"=36 positions) or a sample of transfer function measured at a given position of the stirrer ("1POS").

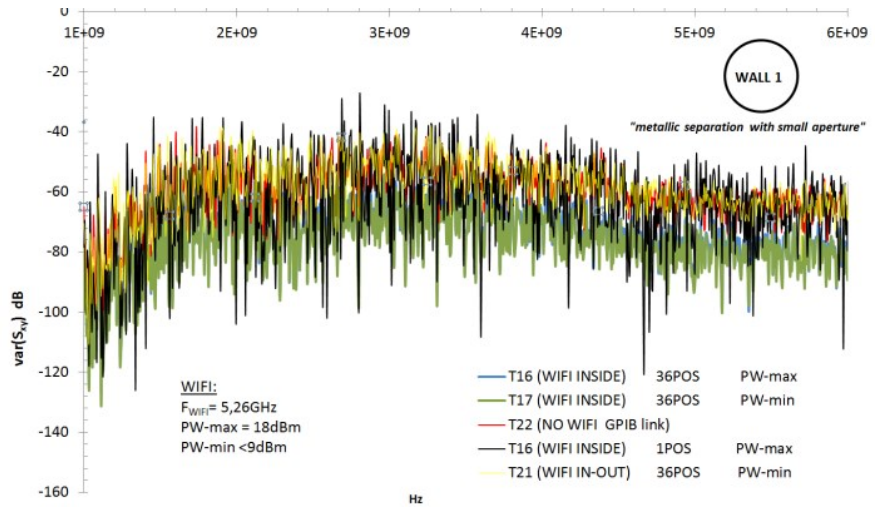

Figure 7 : TF measurement between the two cavivities for various localisations of the WIFI antennas and stirrer position numbers

First of all, in all configurations the transfer function has the same overall variation versus frequency which is explained by the fact that the hole on the separating wall between the 2 volumes behaves as a high pass filter whose cut-off frequency can be deduced from the geometrical shape and dimensions of the hole. Above the cut-off frequency the signal is driven by 
the various dissipative mechanisms (losses and EM leaks) inside and between both volumes.

Moreover one can note that on one hand, the levels obtained on the mean transfer function in some cases of WIFI communication link are lower than the one obtained with the reference configuration. This can be explained by the fact that some measurements of transfer functions at some specific positions of the stirrer during the rotation are corrupted due to an interruption of the WIFI link as demonstrated in the power spectrum measurement in Figure 6. Only not-corrupted signals for specific positions have been acquired. Increasing the power of the WIFI system up to its maximum did not enable to recover the TF signals for all stirrer positions. Nevertheless, when the WIFI communication link is active and operates properly, we can see that the transfer function at these given positions of the stirrer is correctly measured and gives anyway a good estimation of the TF.

Consequently, the stirring process inside these volumes enhances the performances of the WIFI link since for given positions of WIFI antennas, it will always be possible to acquire some data. However, in this case, the statistical postprocessing of the measurement will need to take into account the interruption of the WIFI link in order to correctly extract the mean transfer function over the stirrer rotations. In addition, because of the existence of corrupted TF signals at specific stirrer positions it might be interesting to investigate if number of stirrer positions larger than usual MSRC measurements applied in EMC may enhance the transmission. In addition, one might think of studying the influence of applying a stirring in both emitting and receiving volumes.

Note that the communication still works in IN-OUT configuration even if, in the experiment, we can consider that the structure is mechanically and "electromagnetically" closed. For opened structures (with holes to the outside); is it likely that the results might be more reliable. This is of course is interesting for future applications which intend to use this experimental technique to characterize propagation channels in real vehicles or ground installations for which openings generally allow good transmission of signals in the WIFI operating frequency band.

\section{CONCLUSION}

In this paper, we have shown how an experimental set-up using WIFI communication and mode stirring in a reverberating environment can be combined in order to characterize a propagation channel in closed and confined enclosures and to evaluate at the same time the integrity of a wireless communication signal.

Nevertheless, in order to apply such techniques in real structures (such as vehicles or ground installations) further investigations are needed to analyze how these conclusions can be extended to high-losses enclosures. In addition, as far as critical functions are concerned such techniques can be used to evaluate the security of wireless links towards the exterior of structures as well as their susceptibility to intentional or nonintentional electromagnetic interferences.
[1] Can Wireless Power Transfer Benefit from Large Transmitter Arrays? Salil Kashyap, Emil Bj "ornson and Erik G. Larsson, IEE 2015

[2] IEE 802.11a/g, IEEE Wireless Local Area Networks

[3] Improving Wireless Power Transmission Efficiency Using Chaotic, Ana Collado, and Apostolos Georgiadis IEE 2012

[4] Assessing the performance of ZigBee in a reverberant environment using a mode stirred chamber

[5] I.Junqua, F.Issac, C.Fiachetti ; Bas Michielsen, « Relation entre les écarts types des paramètres[S] d'un équipement dans une CRBM », CEM2002, Grenoble.

[6] I Junqua, High frequency coupling mechanisms in complex systems: analysis and assessment by the PoWer Balance method, Ph.D. Dissertation of Lille University, June 2010 (in French) 